\title{
Methyl- $\beta$-Cyclodextrins Preferentially Remove Cholesterol from the Liquid Disordered Phase in Giant Unilamellar Vesicles
}

\author{
Susana A. Sanchez • German Gunther • \\ Maria A. Tricerri · Enrico Gratton
}

Received: 22 December 2010/Accepted: 14 February 2011/Published online: 6 April 2011

(C) The Author(s) 2011. This article is published with open access at Springerlink.com

\begin{abstract}
Methyl- $\beta$-cyclodextrins (M $\beta \mathrm{CDs})$ are molecules that are extensively used to remove and to load cholesterol (Chol) from artificial and natural membranes; however, the mechanism of Chol extraction by $\mathrm{M} \beta \mathrm{CD}$ from pure lipids or from complex mixtures is not fully understood. One of the outstanding questions in this field is the capability of $\mathrm{M} \beta \mathrm{CD}$ to remove Chol from lipid domains having different packing. Here, we investigated the specificity of $\mathrm{M} \beta \mathrm{CD}$ to remove Chol from coexisting macrodomains with different lipid packing. We used giant unilamellar vesicles (GUVs) made of 1,2-dioleoylphosphatidylcholine:1,2-dipalmitoylphatidylcholine:free cholesterol, 1:1:1 molar ratio at $27^{\circ} \mathrm{C}$. Under these conditions, individual GUVs present Chol distributed into $l_{o}$ and $l_{d}$ phases. The two phases can be distinguished and visualized using Laurdan generalized polarization and two-photon excitation fluorescence microscopy. Our data indicate that $\mathrm{M} \beta \mathrm{CD}$ removes Chol preferentially from the more
\end{abstract}

S. A. Sanchez $(\bowtie) \cdot$ E. Gratton

Laboratory for Fluorescence Dynamics, University of California at Irvine, Irvine, CA, USA

e-mail: susanas@uci.edu

\section{S. A. Sanchez}

Microscopy and Dynamic Imaging Unit, Fundacion

CNIC-Carlos III, Centro Nacional de Investigaciones

Cardiovasculares, Madrid, Spain

G. Gunther

Laboratorio de Cinética y Fotoquímica, Facultad de Ciencias Químicas y Farmacéuticas, Universidad de Chile, Santiago,

Chile

M. A. Tricerri

Instituto de Investigaciones Bioquímicas de La Plata (INIBIOLP), Fac. Cs. Médicas, Universidad Nacional de La Plata, La Plata, Argentina disordered phase. The process of selective Chol removal is dependent on the $\mathrm{M} \beta \mathrm{CD}$ concentration. At high concentrations, $\mathrm{M} \beta \mathrm{CD}$ also removes phospholipids.

Keywords $\mathrm{M} \beta \mathrm{CD} \cdot$ Cholesterol · GUV $\cdot$ Lipid mixture

\section{Introduction}

Cyclodextrins (CDs) are a family of cyclic oligomers of glucose which have a polar surface and a hydrophobic cavity with a diameter of 5-8 $\AA$ that can accommodate small hydrophobic molecules. CDs are named according to the number of glucose units present in their structure: $\alpha, \beta$ or $\gamma$-CD if they contain six, seven or eight sucrose rings, respectively (Besenicar et al. 2008; Choi et al. 2001; Ohvo and Slotte 1996; Roux et al. 2002). CDs have been used in fundamental research, pharmaceuticals/cosmetics, the food industry and technological applications (Duchene and Wouessidjewe 1992; Hedges 1998; Loftsson and Duchêne 2007).

$\beta$-CD and its derivatives, methyl- $\beta$-CD $(\mathrm{M} \beta \mathrm{CD})$ and 2 -hydroxypropyl- $\beta$-CD, are mostly used in studies concerning cholesterol (Chol) manipulation in membranes (Besenicar et al. 2008). Model systems are used to study the mechanism and the molecular parameters involved in the extraction of Chol by $\mathrm{M} \beta \mathrm{CD}$ from monolayers (Ohvo and Slotte 1996), suspended bilayers (Giocondi et al. 2004), giant unilamellar vesicles (GUVs) made of artificial mixtures (Veatch and Keller 2003) and natural membranes (Bernardino de la Serna et al. 2004). In vivo, manipulation of membrane Chol using $\mathrm{M} \beta \mathrm{CD}$ has become popular as a tool to study the putative Chol-enriched cell membrane domains (Brown 2001; Ilangumaran and Hoessli 1998; Jaureguiberry et al. 2010; Lafont et al. 2002; Zidovetzki and Levitan 2007). Despite all the research performed, the 
mechanism of extraction of Chol by $\mathrm{M} \beta \mathrm{CD}$ is not well understood.

We have shown (Sánchez et al. 2007) that the lipid phase is an important parameter for the extraction of Chol by rHDL, which preferentially removes Chol from $l_{d}$ domains. The phase of the lipids where Chol is immersed appears to be an important parameter for Chol extraction from the bilayer. In this article, the role of the lipid phase in the extraction of Chol by $\mathrm{M} \beta \mathrm{CD}$ will be studied.

Lipid mixtures such as 1,2-dioleoyl-sn-glycero-3phosphocholine (DOPC)/1,2-dipalmitoyl-sn-glycero-3-phosphocholine (DPPC)/free cholesterol (FC, 1:1:1) and 1-palmitoyl, 2-oleoyl sn-glycero-3-phosphocholine (POPC)/ sphingomyelin (SM)/FC (6:1:1) have been well characterized and present $l_{o} / l_{d}$ phases that coexist at some specific temperatures (Sánchez et al. 2007; Veatch et al. 2004). In cuvette studies, while using either small or large unilamellar vesicles of these mixtures, it is likely that all vesicles present domain separation at the correct temperature. However, the registered signal averages the information from the two phases.

The study of the specific interaction of a compound with different lipid domains requires (1) single liposome resolution, (2) spatial resolution and (3) a property that can distinguish the different phases in a bilayer. Our methodology fulfills these three requirements. The size of the GUVs, 10-100 $\mu \mathrm{m}$, offers a bilayer surface large enough to visualize within a single liposome under the microscope (spatial resolution) lipid domains with different packing, based on their water content (Laurdan generalized polarization [GP] measurements).

Laurdan GP measurements are based on the shift of the emission spectra of the fluorescent dye Laurdan when the water content in the bilayer changes (Parasassi et al. 1994; Sánchez et al. 2007). For GUVs of POPC and Chol, GP changes can be directly used to quantify Chol content (Sánchez et al. 2007). For ternary mixtures presenting phase separation, the direction and magnitude of the GP changes provide information about the Chol content in the different phases (Bagatolli et al. 2003; Sánchez et al. 2007; Tricerri et al. 2005).

Here, we show the preferential removal of Chol mediated by $\mathrm{M} \beta \mathrm{CD}$ from bilayers containing phase coexistence. Our results on the interaction of $\mathrm{M} \beta \mathrm{CD}$ with GUVs made of DOPC/DPPC/FC $1: 1: 1$ at $25^{\circ} \mathrm{C}$ show that $\mathrm{M} \beta \mathrm{CD}$ removes Chol preferentially from the $l_{d}$ phase.

\section{Materials and Methods}

Materials

FC and phospholipids were obtained from Avanti Polar Lipids (Alabaster, AL) and used without further purification.
Laurdan, 6-lauroyl-2-(dimethylamino) naphthalene, was purchased from Molecular Probes (Eugene, OR). Tris (hydroxymethyl) aminomethane hydrochloride (Trizma $\mathrm{HCl}$ ) and $\mathrm{M} \beta \mathrm{CD}$ were from Sigma-Aldrich (St. Louis, MO).

\section{Methods}

\section{GUV Formation}

The protocol to generate GUVs is a modified version of the method published by Angelova and coworkers (Angelova and Dimitrov 1986; Angelova et al. 1992). Briefly, stock solutions of phospholipids were prepared in chloroform at a concentration of $0.2 \mathrm{mg} / \mathrm{ml}$. Two microliters of the stock were added to each platinum wire of the growing chamber and dried under $\mathrm{N}_{2}$ for about $30 \mathrm{~min}$ to remove any traces of the remaining solvent. The chamber was sealed with a coverslip and thermostatted with a water-circulating bath to the growing temperature (normally $10^{\circ} \mathrm{C}$ over the corresponding transition temperature of the lipid). Immediately before connecting the $\mathrm{Pt}$ wires to the function generator (model 3035B; Hewlett-Packard, Santa Clara, CA), 1 mM Tris buffer, $\mathrm{pH} 8.0$ (previously warmed up to the growing temperature), was added to the chamber. Temperature was monitored by a digital thermocouple (model 400B; Omega, Stamford, $\mathrm{CT}$ ) in contact with the wires. A low-frequency AC field (frequency $10 \mathrm{~Hz}$, amplitude $2 \mathrm{~V}$ ) was applied to the $\mathrm{Pt}$ wires in order to grow the vesicles by the electroformation method. A CCD color video camera (CCD-Iris; Sony, New York, $\mathrm{NY}$ ), attached to the microscope allowed visualization of vesicle formation and selection of the target GUV.

\section{Laurdan GP and Imaging}

Laurdan is used as a membrane fluorescent probe because of its large excited state dipole moment, which results in its ability to report the extent of water penetration into the bilayer surface due to the dipolar relaxation effect (Weber and Farris 1979). Water penetration has been correlated with lipid packing and membrane fluidity (Parasassi and Gratton 1995; Parasassi et al. 1991). The emission spectrum of Laurdan in a single phospholipid bilayer is centered at $440 \mathrm{~nm}$ when the membrane is in the gel phase and at $490 \mathrm{~nm}$ when solubilized in the liquid crystalline phase. In addition, its emission is minimal in an aqueous environment. The GP function gives a mathematically convenient and quantitative way to measure the emission shift. The function is given by

$G P=\frac{I_{440}-I_{490}}{I_{440}+I_{490}}$

where $I_{440}$ and $I_{490}$ are the emission intensities at 440 and $490 \mathrm{~nm}$, respectively. A full discussion on the use and 
mathematical significance of GP can be found in the literature (Bagatolli et al. 2003; Parasassi and Gratton 1995). If Laurdan is in a phospholipid environment, such as a liposome, its spectrum will move according to the water content of the bilayer. The fluorescence emission of Laurdan at the fluid-ordered bilayer is blue-shifted compared with the emission from the fluid-disorder, allowing identification of the phase of the lipid domain directly from the GP image. To calculate the GP value in a two-photon microscope, an excitation wavelength of $780 \mathrm{~nm}$ and a two-channel system with the corresponding filters on the emission is used (see below for details).

\section{Experimental Setup}

Images were collected on a scanning two-photon fluorescence microscope designed at the Laboratory for Fluorescence Dynamics (So et al. 1995, 1996). The LD-Achroplan $20 \times$ long working distance air objective with N.A. 0.4 (Zeiss, Holmdale, NJ) was used. A mode-locked titaniumsapphire laser (Mira 900; Coherent, Palo Alto, CA) pumped by a frequency-doubled Nd: vanadate laser (Verdi, Coherent) and set to $780 \mathrm{~nm}$ was used as the two-photon excitation light source. A galvanometer-driven $x-y$ scanner was positioned in the excitation path (Cambridge Technology, Watertown, MA) to achieve beam scanning in both the $x$ and $y$ directions. A quarter wave-plate (CVI Laser, Albuquerque, NM) was aligned and placed in the path of the beam to minimize the polarization effects of the excitation light. Fluorescence emission was observed through a 350-600 nm band-pass filter (BG39 filter; Chroma Technology, Brattleboro, VT). A miniature photomultiplier (R5600-P; Hamamatsu, Bridgewater, NJ) was used for light detection in the photon counting mode, and counts were acquired using an ISS card (ISS, Inc., Champaign, IL). A two-channel detection system was attached for GP function image collection. The fluorescence was split in two using a Chroma Technology 470DCXR-BS dichroic beam splitter in the emission path. Interference filters, Ealing 490 and Ealing 440, were placed in the emission paths to further isolate the two areas of the Laurdan emission spectrum, respectively. Separate detectors were used for each channel to simultaneously collect the 490 and $440 \mathrm{~nm}$ emissions. The two images were simultaneously collected and then recombined to form the GP image of the sample using the data-acquisition section of the SimFCS program (Laboratory for Fluorescence Dynamics, Irvine, CA). Corrections for the wavelength dependence of the emission detection system were made through comparison of the GP value of a known solution (Laurdan in dimethyl sulfoxide [DMSO]) (Bagatolli and Gratton 1999; Sanchez et al. 2002) acquired on an ISS model PC1 steady-state fluorometer and then under the microscope.
GP Data Analysis

Two simultaneous $256 \times 256$ pixel images were obtained from the vesicle (image channel 1 and image channel 2). The two intensity images were used to create a GP image by applying the GP formula (Eq. 1) pixel by pixel. From the resulting GP image a histogram of number of pixels vs. GP was created and the center of the histogram was reported as the average GP value. GP values could range from -1 to +1 according to Eq. 1 , and a color scale was used for visualizing pixels with different GP.

When the GP image presented areas with different GP, both the histogram and the GP image could be deconvoluted in high and low values, in which case the average GP is given by

$\mathrm{GP}_{\mathrm{avg}}=\left(\mathrm{GP}_{1} \times f_{1}\right)+\left(\mathrm{GP}_{2} \times f_{2}\right)$

where $f_{1}$ and $f_{2}$ correspond to the fractional contributions of the components with higher $\left(\mathrm{GP}_{1}\right)$ and lower $\left(\mathrm{GP}_{2}\right) \mathrm{GP}$ values.

\section{GP Kinetics Measurements}

GUVs were grown at $60^{\circ} \mathrm{C}$ in $1 \mathrm{mM}$ Tris, $\mathrm{pH}$ 8.0. The temperature was continuously monitored using a thermocouple attached to platinum wires. Laurdan in DMSO was added to the solution (in a 1:300 Laurdan/lipid molar ratio) and incubated for $15 \mathrm{~min}$. The temperature was slowly decreased to the desired value, and before adding the $\mathrm{M} \beta \mathrm{CD}$, two or three GP images, corresponding to the zero time in the kinetics, were taken. In order to ensure the quick mixing of the Chol acceptor in the solution, 20-30 $\mu \mathrm{l}$ of a concentrated stock solution of $\mathrm{M} \beta \mathrm{CD}$ was diluted in $1 \mathrm{ml}$ of the growing buffer and the solution was placed back in the chamber immediately. After addition of the acceptor, GP images were taken every $15 \min$ for $2 \mathrm{~h}$.

\section{Results}

Interaction of $\mathrm{M} \beta \mathrm{CD}$ with the Lipid Bilayer Does not Produce GP Changes

We previously reported a correlation between the Laurdan GP and Chol contents in the lipid bilayer and the use of this parameter to monitor the kinetics of Chol removal from GUVs (Sánchez et al. 2007). In order to use $M \beta C D$ as a Chol acceptor, we designed a control experiment to estimate the changes in GP value due to the direct interaction of $\mathrm{M} \beta \mathrm{CD}$ with the lipids. Figure 1 shows the GP values for POPC bilayers containing 0 and $30 \%$ Chol before and after $2 \mathrm{~h}$ incubation with $0.8 \mathrm{mM} \mathrm{M} \beta \mathrm{CD}$. No significant GP changes were observed for POPC GUVs after the 


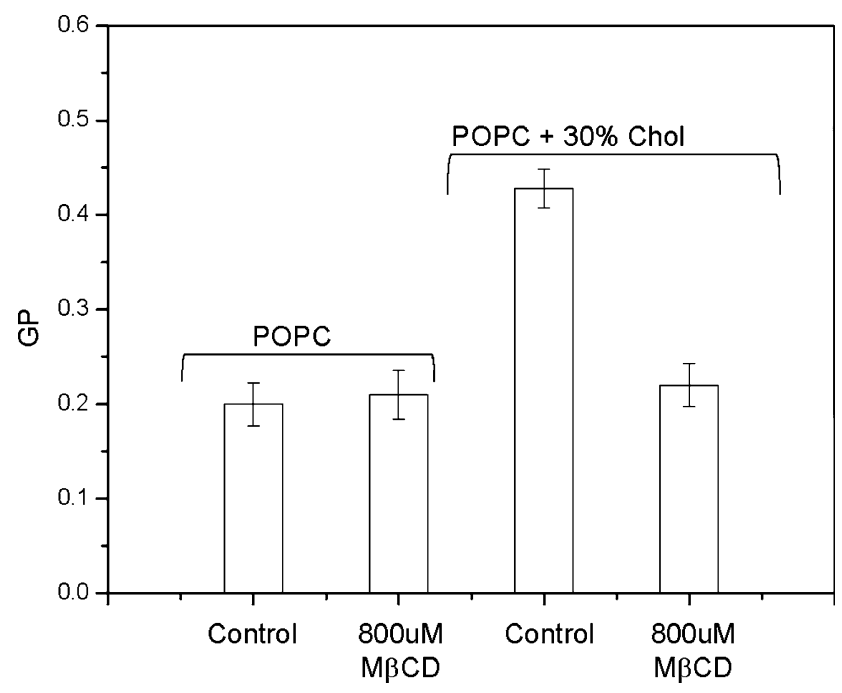

Fig. 1 Changes in Laurdan GP value for GUVs of POPC and POPC plus $33 \%$ molar Chol after incubation for $2 \mathrm{~h}$ with $800 \mu \mathrm{M} \mathrm{M} \beta \mathrm{CD}$. GP value of 10 GUVs before and after the addition was averaged. Working temperature was $25^{\circ} \mathrm{C}$

incubation period with $\mathrm{M} \beta \mathrm{CD}$. Instead, under the same conditions, the GP for GUVs of POPC containing 30\% Chol decreased to the value corresponding to pure POPC. This experiment demonstrates that the changes in GP are in fact due to Chol removal by the $\mathrm{M} \beta \mathrm{CD}$ and not to the interaction of $\mathrm{M} \beta \mathrm{CD}$ with the lipids. Addition of $\mathrm{M} \beta \mathrm{CD}$ $0.8 \mathrm{mM}$ did not produce detectable changes in the volume of the GUV made of POPC plus 30\% Chol (Sánchez et al. 2007). However, the same concentration reduced by $\sim 60 \%$ of the volume of the GUVs made of pure POPC (data not shown) demonstrated the capability of $\mathrm{M} \beta \mathrm{CD}$ to solubilize phospholipids, a process that seems to depend on the presence of Chol. It is important to note that Chol removal could be underestimated in our experiments. This is due to the fact that the platinum wires where the target GUV is attached also contain other GUVs that may replenish some of the Chol being removed by the $\mathrm{M} \beta \mathrm{CD}$.

\section{Interaction of $\mathrm{M} \beta \mathrm{CD}$ with Coexisting $l_{o} / l_{d}$ Phases}

The mixture DOPC/DPPC/FC (1:1:1 molar ratio) presents a homogeneous phase at $37^{\circ} \mathrm{C}$ and coexisting liquid ordered/ liquid disordered $\left(l_{o} / l_{d}\right)$ macrodomains at temperatures below $32^{\circ} \mathrm{C}$. The characteristics of these phases have been described (Veatch et al. 2004; Sánchez et al. 2007). Figure 2a shows the GP image of a GUV at $25^{\circ} \mathrm{C}$; the equatorial cross section shows a bright ring (Laurdan associated to the lipid bilayer) and a dark center (interior of the GUV). The discontinuity of the ring on the left side corresponds to the GUV's attachment to the platinum wire where it has been formed. The lipid bilayer shows two areas with different GP, colored in orange ( $l_{o}$ macrodomain) and green
( $l_{d}$ macrodomain). At $25^{\circ} \mathrm{C}$, the described phase separation was observed in $100 \%$ of the GUVs in all experiments performed. Control experiments (without $\mathrm{M} \beta \mathrm{CD}$ addition) confirmed constant GP and areas for the two phases over a span of $2 \mathrm{~h}$. Using the SimFCS Software, the two phases were separated (Fig. 2b, c) and the number of pixels (size of the domain) and the corresponding GP calculated. Experimentally, the areas corresponding to each phase were determined before the interaction with $\mathrm{M} \beta \mathrm{CD}$, and the two phases presented similar numbers of pixels. However, after $\mathrm{M} \beta \mathrm{CD}$ was added and due to the size changes produced on the phases, determination of the area of each phase became more difficult; therefore, we analyzed the volume of the target GUV as a parameter to follow the interaction together with the GP value.

We determined the selectivity of $\mathrm{M} \beta \mathrm{CD}$ to remove Chol from either the $l_{o}$ or $l_{d}$ macrodomain. The Laurdan GP value is related to the Chol content in the bilayer (Parasassi et al. 1994; Sánchez et al. 2007), and changes in this parameter after adding $\mathrm{M} \beta \mathrm{CD}$ correlate with Chol being removed from the bilayer (Sánchez et al. 2007). The GP values of the coexisting $l_{o} / l_{d}$ phases after the addition of $\mathrm{M} \beta \mathrm{CD}$ at $25^{\circ} \mathrm{C}$ as a function of time are presented in Figs. 3 and 4. A single DOPC/DPPC/FC 1:1:1 vesicle was chosen for each kinetics study. Nevertheless, the data correspond to the general observed behavior. Variations among individual GUVs depend on the size of the liposome and the individual domains. At $25^{\circ} \mathrm{C}$ these GUVs show two macrodomains $\left(l_{o}\right.$ and $\left.l_{d}\right)$, with GP values of 0.49 (orange) and 0.31 (yellow), respectively (Fig. 3a). These GP values correspond to the initial GP $\left(\mathrm{GP}_{\mathrm{i}}\right)$ for each phase before the addition of $\mathrm{M} \beta \mathrm{CD}$, and they are shown as a horizontal solid line in Fig. $3 \mathrm{a}, \mathrm{b} . \mathrm{GP}_{\mathrm{f}}$ indicates the expected final GP values for each phase if all the Chol is removed from the bilayer (Fig. 3a, b). These final values were obtained from GUVs made with the mixture DOPC/ DPPC (1:1). This binary mixture also presents coexisting phases at $25^{\circ} \mathrm{C}$ (Sánchez et al. 2007; Veatch et al. 2004), and the corresponding values for $l_{o}(\mathrm{GP}=0.56)$ and $l_{d}$ $(\mathrm{GP}=0.01)$ are shown as dotted lines in the same plot.

After addition of $\mathrm{M} \beta \mathrm{CD}$, the GP for the phases was measured over a $2 \mathrm{~h}$ period. Figure $3 \mathrm{a}$ shows the changes in GP as a function of time after the addition of $0.25 \mathrm{mM} \mathrm{M} \beta \mathrm{CD}$ to DOPC/DPPC/FC $1: 1: 1 \mathrm{GUVs}$ at $25^{\circ} \mathrm{C}$. This concentration of $\mathrm{M} \beta \mathrm{CD}$ was chosen based on previous results from our group (Sánchez et al. 2007) showing that at $37^{\circ} \mathrm{C}$ the GP of this mixture decreased $60 \%$ from its original value when $2 \mathrm{mM} \mathrm{M} \beta \mathrm{CD}$ is used. At this temperature the GP value is homogeneous, presenting no macrophase separation (Sánchez et al. 2007; Veatch et al. 2004). The upper panel of Fig. 3a shows the image of the target GUV at time zero and after $110 \mathrm{~min}$ of incubation with $\mathrm{M} \beta \mathrm{CD}$. The dotted circular line around the GUV 


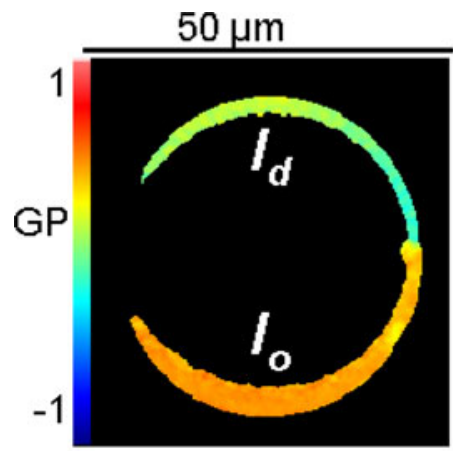

A

Fig. 2 GP image analysis. a Laurdan GP image (equatorial $z$-section) of a GUV made of DOPC/DPPC/FC 1:1:1 at $25^{\circ} \mathrm{C}$ showing phase separation, $l_{o}$ (orange) and $l_{d}$ (green). The SimFCS program was used to


Fig. 3 GP changes of coexisting $l_{o} / l_{d}$ during interaction with $0.25 \mathrm{mM}$ (a) and $1 \mathrm{mM}(\mathbf{b}) \mathrm{M} \beta \mathrm{CD}$. Continuous lines at $\mathrm{GP}_{\mathrm{i}}$ correspond to the $\mathrm{GP}$ value of the $l_{o}$ and $l_{d}$ domains in the DOPC/DPPC/FC (1:1:1 mol \%) mixture and are the initial values for the kinetics. Dotted lines at $\mathrm{GP}_{\mathrm{f}}$ are the GP values for the coexisting $l_{o}$ and $l_{d}$ phases in DOPC/DPPC (1:1) and correspond to the expected final GP values if all $\mathrm{Chol}$ is removed (Sánchez et al. 2007). Arrows represent the expected magnitude and direction for the GP changes when Chol is removed from the domains.

indicates that no changes in the GUV radius were detected. The plots in Fig. 3a show the GP (circles) and volume (squares) changes during the incubation time. The GP for the $l_{o}$ phase (closed circles) did not change significantly during the observation period; instead, the GP of the $l_{d}$

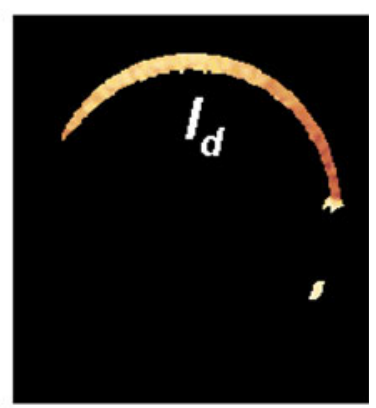

C

analyze the GP image. Pixels with different GP values can be separated and the individual $l_{o}(\mathbf{b})$ and $l_{d}(\mathbf{c})$ phases followed in time. The number of pixels is related to the area of each phase (Color figure online)
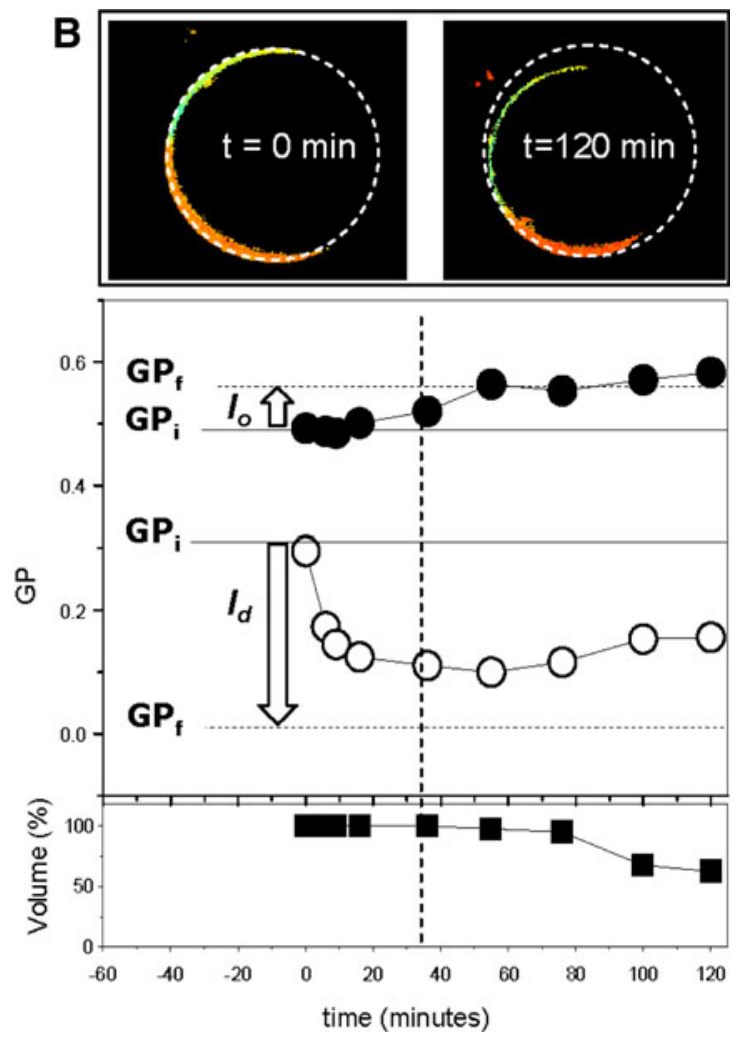

Vertical dashed line at $45 \mathrm{~min}$ in $\mathbf{b}$ indicates the beginning of GUV shrinkage. The GP changes of the $l_{o}$ (closed circles) and $l_{d}$ (open circles) domains and volume reduction of the GUV (open squares) were followed for $2 \mathrm{~h}$ at $25^{\circ} \mathrm{C}$. Images on top of the plots are GP images at the beginning ( $0 \mathrm{~min})$ and end (110 and $120 \mathrm{~min})$ of the incubation time, and the circular dotted line around the GUVs indicates the original size and shows the decrease in volume experimented by the target GUV (Color figure online)

phase decreased $\sim 33 \%$ from the initial value of 0.31 , indicating partial removal of Chol from this phase. No changes in the volume (calculated from changes in ratio) of the GUV were observed during the incubation time, as shown in the bottom plot (closed squares). 
A
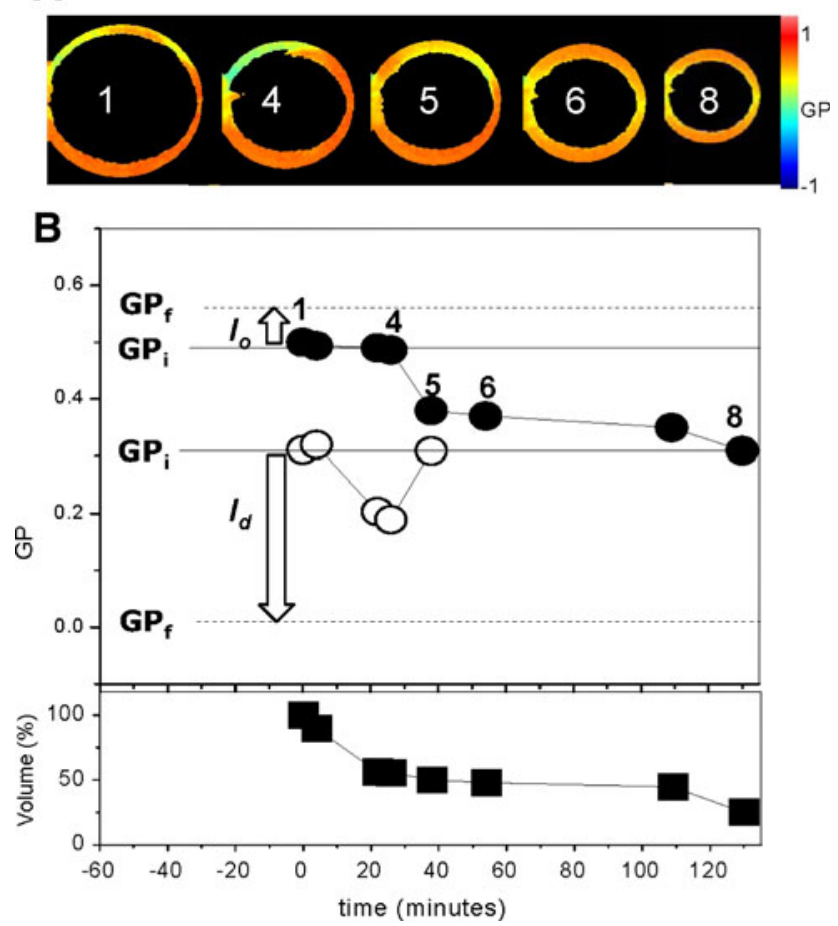

Fig. 4 GP changes of coexisting $l_{o} l_{d}$ during interaction with $2 \mathrm{mM} \mathrm{M} \beta \mathrm{CD} . \mathrm{GP}_{\mathrm{i}}, \mathrm{GP}_{\mathrm{f}}$, arrows and symbols have the same meaning as in Fig. 3. Images on top of the plots are GP images at different points during the incubation time, and the circular dotted line around the GUVs indicates the original size. Numbers at the center of the GUVs in the GP images correspond to the experimental points shown in the plot (Color figure online)

Figure $3 \mathrm{~b}$ shows the changes after incubation with $1 \mathrm{mM} \mathrm{M} \beta \mathrm{CD}$. The comparison of the initial $(t=0 \mathrm{~min})$ and final $(t=120 \mathrm{~min})$ GP images of the target GUV clearly show a decrease in the size of the GUV (top panel in Fig. 3b). The GP changes (circles) during the first $20 \mathrm{~min}$ follow a trend similar to that observed using $0.25 \mathrm{mM} \mathrm{M} \beta \mathrm{CD}$ : The GP of the $l_{o}$ phase (closed circles) does not change significantly, and the GP of the $l_{d}$ phase (open circles) decreases by $66 \%$ from the original value, indicating very efficient Chol removal. This removal curve could be fitted to a single exponential with a $t_{1 / 2}=$ $5.6 \pm 0.4 \mathrm{~min}$. Approximately $30 \mathrm{~min}$ later, the GP value for the $l_{o}$ phase starts increasing toward the value calculated for the $l_{o}$ phase without Chol, suggesting that at longer incubation times, Chol could be removed from the $l_{o}$ phase. However, at this time, the size of the GUV starts to decrease (closed squares), making the interpretation more complex.

A further increase of the $\mathrm{M} \beta \mathrm{CD}$ concentration makes the effects more dramatic. Figure 4 shows the results after addition of $2 \mathrm{mM} \mathrm{M} \beta \mathrm{CD}$. The GP changes during the first $25 \mathrm{~min}$ are similar to those observed after the addition of $1 \mathrm{mM} \mathrm{M} \beta \mathrm{CD}$ : fast decrease of GP of the $l_{d}$ phase (open circles) and no significant change of the GP of the $l_{o}$ phase (closed circles). Instead, volume reduction starts immediately after the addition. After $25 \mathrm{~min}$, the GUV is $50 \%$ of its original volume (closed squares). From 25 to $110 \mathrm{~min}$, the size of the GUV remained unchanged but the phase separation disappeared and the GUV presented just one GP value (image 6). By the end of the incubation period there was a further decrease in volume, and the final GUV was reduced by $78 \%$ from the original size (Fig. 4, image 8). After the severe shrinkage and disappearance of the phases, interpretation of the GP (as a reporter of the Chol content) involves a combination of factors difficult to analyze separately. It is clear, however, that at this concentration, for this particular system, $\mathrm{M} \beta \mathrm{CD}$ induced dramatic deformations. It is important to mention that at this high concentration of $\mathrm{M} \beta \mathrm{CD}(2 \mathrm{mM})$ some of the volume reduction could be attributed to osmolarity changes; however, the preferential disappearance of the $l_{d}$ phase shows that the effect of $\mathrm{M} \beta \mathrm{CD}$ is more specific for this type of domain.

Interaction of $\mathrm{M} \beta \mathrm{CD}$ with Independent $l_{o}$ and $l_{d}$ Phases

As indicated by the experiments shown above, a decrease in the GP value of the $l_{d}$ phase together with no changes in the GP value of the $l_{o}$ phase in the $l_{d} l_{d}$ coexisting system indicates that $\mathrm{M} \beta \mathrm{CD}$ removes preferentially Chol from the $l_{d}$ phase These results can also be explained if Chol is removed from the $l_{o}$ phase and, in order to restore the equilibrium, Chol from the neighbor $l_{d}$ phase moves to the $l_{o}$ phase. In order to test this hypothesis, we performed the experiments on two separated phases. The composition of the $l_{o}$ and $l_{d}$ phases coexisting at $25^{\circ} \mathrm{C}$ in the DOPC/DPPC/ FC (1:1:1) mixture has been determined (Veatch et al. 2004): The $l_{o}$ phase is composed of DOPC/DPPC/FC (5:53:42 mol\%) and the $l_{d}$ phase by DOPC/DPPC/FC (60:18:22 mol\%). GUVs made with the two mixtures were independently grown, and the changes in GP and morphology after addition of $1 \mathrm{mM} \mathrm{M} \beta \mathrm{CD}$ were followed for a period of $100 \mathrm{~min}$. Figure 5a shows the GP changes on the $l_{d}$ phase (DOPC/DPPC/FC, 60:18:22 mol\%) after addition of $1 \mathrm{mM} \mathrm{M} \beta \mathrm{CD}$. The images show the GUV at $t=0$ and at the end of the incubation period $(t=100 \mathrm{~min})$. The dotted circle around the GUV indicates that the size of the GUV has decreased by the end of the experiment. The GP starts to decrease immediately after the addition of $\mathrm{M} \beta \mathrm{CD}$, reaching a value of -0.13 by $20 \mathrm{~min}$. The kinetics of the removal fits to a single exponential decay, with $t_{1 / 2}=7.7 \pm 0.6 \mathrm{~min}$. The GUV volume started to decrease around 6 min after the addition of CD. By 20 min (dotted vertical line) the GUV volume was reduced by $40 \%$ of its original size and remained unchanged until the end of the observation period (100 min). 




B

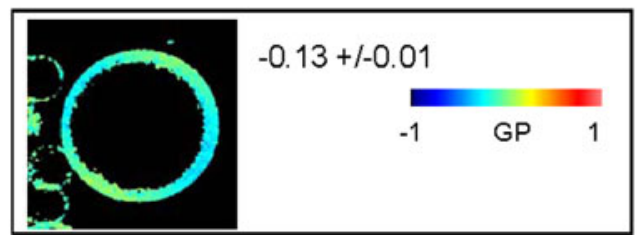

Fig. 5 Kinetics for the separated $l_{o}$ and $l_{d}$ phases after addition of $1 \mathrm{mM} \mathrm{M} \beta \mathrm{CD}$. a Changes in GP value (open circles) and volume (closed squares) for the separated $l_{d}$ phase (DOPC/DPPC/FC, 60:18:22 mol\%). Comparison of GUV images at $t=0$ and $t=100$ min shows the decrease in size at the end of kinetics (dotted circular white line around the GUV on the image at $t=100$, plot (a). b GUV of the $l_{d}$ mixture without Chol (DOPC/DPPC/FC 77:23:0 mol\%) with the corresponding average GP value for the homogeneous phase calculated from five independent GUVs.

To characterize the expected morphology and GP value after all the Chol was removed from this mixture, we constructed a GUV of the $l_{o}$ mixture without Chol (DOPC/ DPPC/FC, 77:23:0 mol\%). The GP image is shown in Fig. 5b: a GUV with a homogeneous GP value around -0.13 . This is the same GP value presented at the end of the kinetics in Fig. 5a, indicating that $1 \mathrm{mM} \mathrm{M} \beta \mathrm{CD}$ removed all the Chol from the separated grown $l_{d}$ phase.

In Fig. $5 \mathrm{c}$, the results for the separated $l_{o}$ phase (DOPC/ DPPC/FC, 5:53:42 mol\%) are shown. No changes in GP (closed circles) and volume (closed squares) were observed during the $2 \mathrm{~h}$ incubation with $1 \mathrm{mM} \mathrm{M} \beta \mathrm{CD}$. Figure $5 \mathrm{~d}$ shows the center and top images of a GUV made with the mixture without Chol (DOPC/DPPC/FC 9:91:0 mol\%). This GUV shows the final GP and morphology expected if all the Chol is removed from the $l_{o}$ phase. The mixture without Chol shows phase separation at $25^{\circ} \mathrm{C}$, and the GP values for each phase are shown in Fig. $5 \mathrm{~d}$. The absence of GP changes and the lack of phase separation (Fig. 5c) after incubation of the separated $l_{o}$ phase with $\mathrm{M} \beta \mathrm{CD}$ indicate that no Chol is removed from this GUV.
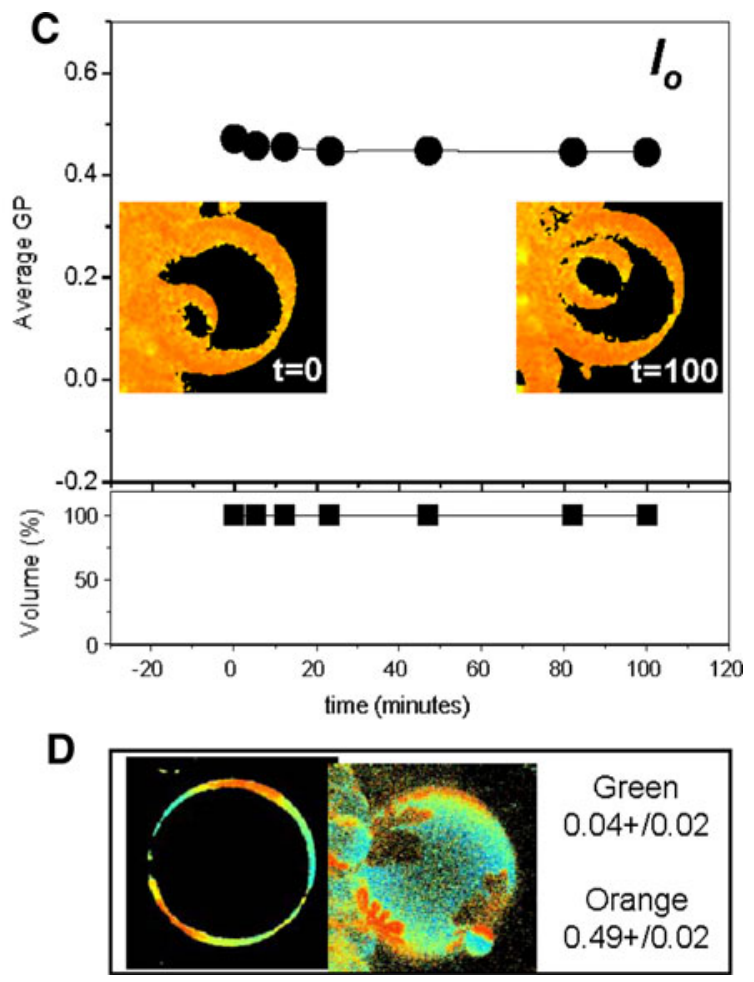

c Changes in GP value (closed circles) and volume (closed squares) for the separated $l_{o}$ phase (DOPC/DPPC/FC, 5:53:42 mol\%). Images of the GUV at $t=0$ and $t=100 \mathrm{~min}$ show no changes in size during the kinetics. d Center and top views of a GUV of the $l_{o}$ mixture without Chol (DOPC/DPPC/FC 9:91:0 mol\%) showing phase separation. GP values for the different phases (green and orange) are given as an average of four GUVs. The same color scale was used for all images. Temperature $25^{\circ} \mathrm{C}$ (Color figure online)

\section{Discussion and Conclusions}

Our experimental approach correlates the changes in Laurdan GP value with changes in Chol content in the membrane (Parasassi et al. 1994; Sánchez et al. 2007). The measured GP value decreased in the $l_{d}$ phase as a result of the interaction with $\mathrm{M} \beta \mathrm{CD}$. This change can be due to the direct removal of Chol from the $l_{d}$ phase or to a rapid reequilibration of Chol after being solubilized from the $l_{o}$ phase. In our previous work, using rHDL (Sánchez et al. 2007), we proposed that Chol could diffuse between the phases to reequilibrate after extraction. In the case of $\mathrm{M} \beta \mathrm{CD}$, we have more information. When $\mathrm{M} \beta \mathrm{CD}$ interacted with phases in the same GUV (Fig. 5), no Chol was solubilized from the $l_{o}$ phase and $\sim 100 \%$ of the Chol was removed from the $l_{d}$ phase. Interestingly, the rates of decrease in GP in the $l_{d}$ phase when coexisting with the $l_{o}$ phase $(5.6 \pm 0.4 \mathrm{~min})$ or when separated $(7.7 \pm 0.6 \mathrm{~min})$ were similar. These experiments support the interpretation that the decrease in GP values observed in the $l_{d}$ phase during the interaction with $\mathrm{M} \beta \mathrm{CD}$ is due to preferential Chol removal from the $l_{d}$ phase. 


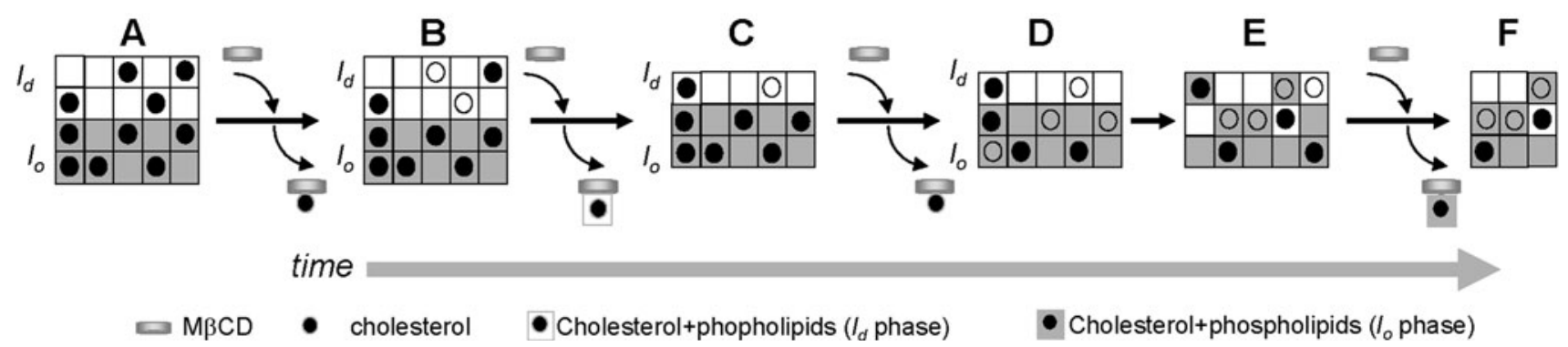

Fig. 6 Model for the interaction of $\mathrm{M} \beta \mathrm{CD}$ and membranes presenting phase separation. Empty circles symbolize Chol removed by $\mathrm{M} \beta \mathrm{CD}$

The capability of different acceptors to remove Chol from the membranes depends on what has been defined as "cholesterol activity," the tendency of molecules to "escape" from the surface of the bilayer. This tendency, among others, will depend on the entropy-driven tendency of Chol to diffuse. When Chol is solubilized in a mixture of lipids, it segregates in different domains according to the melting temperature $(\mathrm{Tm})$ and chemical nature of the lipids; thus, Chol will be distributed according to the relative affinity for each phase (Veatch et al. 2004). Chol activity in the more fluid phase (mainly formed by unsaturated phospholipids) will be higher than its activity in the more organized phase (containing mainly saturated phospholipids). Our results agree with this concept. M $\beta C D$ preferentially removed Chol from the disordered (i.e., nonraft) phase, the phase where Chol activity is higher.

A molecular explanation for this preference can be found by analyzing the structure of the lipids coexisting in the phases; the unsaturations present in the acyl chains of phospholipids such as DOPC introduces kinks into the structure that limit the ability of Chol to order, to mix homogeneously and to reduce interfacial (Brown 1998) and in-plane (Needham et al. 1988) elasticity. These kinks in the lipids forming the $l_{d}$ phases prevent the deep penetration that is possible with the rigid acyl chains of DPPC. Chol will thus locate more superficially and desorb more easily from the membrane to reach the hydrophobic center in the $\mathrm{M} \beta \mathrm{CD}$ molecules. On the other side, for phases formed mainly by saturated phospholipids such as DPPC, ${ }^{2} \mathrm{H}-\mathrm{NMR}$ data suggest that the hydrophobic thickness of the lipid bilayer is much larger than the $20 \AA$ of the Chol molecule but that the average acyl chain length, in each leaflet, is significantly shorter than the length of Chol (Sankaram and Thompson 1990). Thus, Chol molecules locate part of their tail in the adjacent monolayer (Sankaram and Thompson 1991), allowing the formation of tail-to-tail dimers (Harris et al. 1995), which could impede their desorption, resulting in reduced Chol transfers to the acceptor.

The observed reduction in the GUV volume during the interaction with $\mathrm{M} \beta \mathrm{CD}$ gives some information about the ability of $\mathrm{M} \beta \mathrm{CD}$ to remove phospholipids from the membrane. Theoretical calculations are a good approximation of the expected changes in volume when all the Chol is removed from a GUV composed of DOPC/DPPC/FC (1:1:1 molar ratio). Using the reported molecular areas for the lipids $\left(\sim 70\right.$ and $60 \AA^{2}$ for unsaturated and saturated phospholipids and $\sim 40 \AA^{2}$ for Chol [Evans et al. 1987]) it can be calculated a reduction of $23 \%$ on the surface area if all the Chol is removed from a spherical liposome of a given radius. This reduction in surface area will show a $\sim 35 \%$ decrease in volume (this calculation assumes similar surface areas for the lipids in $l_{o}$ and $l_{d}$ phases). In the interaction of DOPC/DPPC/FC (1:1:1 molar ratio) with $1 \mathrm{mM} \mathrm{M} \beta \mathrm{CD}$, the observed decrease of $\sim 40 \%$ in volume (Fig. 3) may be mainly interpreted as Chol being removed from the GUV; however, in the presence of $2 \mathrm{mM} \mathrm{M} \beta \mathrm{CD}$, the $76 \%$ reduction in volume (Fig. 4) can be explained only if both Chol and phospholipids are removed. Interestingly, in our experiments, a decrease in size was observed for pure POPC GUVs when incubated with $0.8 \mathrm{mM} \mathrm{M} \beta \mathrm{CD}$. However, the presence of $30 \%$ Chol avoided such an effect. More detailed studies are needed to understand the mechanism behind this interaction. Our results clearly indicate that $\mathrm{M} \beta \mathrm{CD}$ is able to remove phospholipids and that this process depends on the presence of Chol.

The fact that $\mathrm{M} \beta \mathrm{CD}$ can remove both Chol and phospholipids with high efficiency and fast kinetics introduces the possibility of a certain degree of toxicity if the same process happens in cellular systems. In artificial systems such as the GUVs, the removal of lipids can be evidenced as a reduction in volume. However, in cells, membrane rearrangement-destruction can induce cell death or hemolysis (Irie et al. 1982).

Figure 6 illustrates a model to explain the molecular events underlying the results presented in Figs. 3 and 4. (A) We have represented the initial state for DOPC/DPPC/ FC $(1: 1: 1)$ at $25^{\circ} \mathrm{C}$ as the $l_{o}$ and $l_{d}$ macrophases coexisting and assuming the same size for each phase for didactic purpose. Chol partitions at $42 \%$ in the $l_{o}$ and $22 \%$ in the $l_{d}$ phase according to the literature (Veatch et al. 2004). (B) At short incubation times and low concentration of $\mathrm{M} \beta \mathrm{CD}$, Chol is mainly removed from the $l_{d}$ phase, leaving an $l_{d}$ phase with less Chol than the initial one and reported by a lower GP value. (C) If the concentration of $\mathrm{M} \beta \mathrm{CD}$ is 
high $(2 \mathrm{mM})$, phospholipids are removed together with Chol from the $l_{d}$ phase, decreasing its size (Fig. 4) and consequently the liposome cross section radius. (D) At longer incubation times, Chol and phospholipids may be removed from the $l_{o}$ phase depending on the $\mathrm{M} \beta \mathrm{CD}$ concentration. (E) Drastic lipid removal induces maximal superficial contact between the phases, promoting phase fusion. (F) From the new phase generated (with an average GP value) $\mathrm{M} \beta C \mathrm{CD}$ can remove $\mathrm{Chol}$ and phospholipids with a consequent decrease in the size of the entire liposome.

The experiments presented here show that $\mathrm{M} \beta \mathrm{CD}$ in a range of concentrations $(0.25-2 \mathrm{mM})$ preferentially and efficiently removes Chol from the $l_{d}$ phase. The same result was found for rHDL (Sánchez et al. 2007): preferential removal of Chol from the $l_{o}$ coexisting phase in two different systems (DOPC/DPPC/FC 1:1:1 and POPC/SM/FC 6:1:1). Further studies will be needed to determine whether rHDL and $\mathrm{M} \beta \mathrm{CD}$ share similar passive mechanisms. The experimental approach presented here could be of importance for applications aiming to transport Chol or other lipids from artificial or natural systems.

Finally, if nanometer-size rafts exist in vivo, we should be cautious in extrapolating our results obtained on large macroscopic domains to the raft case. We will need further studies on the effect of domain size on the Chol removal process. Larger contact areas between the phases may change the "activity" of Chol molecules, causing reequilibration between phases after Chol extraction and the kinetics of the whole process. In addition, different passive and active events, such as lipid composition, protein signaling and the interaction of the membrane components with the cytoskeleton, are specific of each type of cell (Jaureguiberry et al. 2010; Jouni et al. 2002; Sánchez et al. 2010; Zidovetzki and Levitan 2007), keeping domain organization and viability; thus, extrapolation to in vivo systems should be done considering each case.

Acknowledgement S. A. S. and E. G. acknowledge support of the National Institutes of Health (grant PHS 5 P41 RR-03155). S. A. S. and M. A. T. acknowledge support from FABLS, 2008. G. G. acknowledges support from Fondecyt (grant 1080412). M. A. T. acknowledges support from ANPCyT (grants 14443, 26228 and 2106/2008) and CONICET (grant 00953).

Open Access This article is distributed under the terms of the Creative Commons Attribution Noncommercial License which permits any noncommercial use, distribution, and reproduction in any medium, provided the original author(s) and source are credited.

\section{References}

Angelova MI, Dimitrov DS (1986) Liposome electroformation. Faraday Discuss Chem Soc 81:303-311
Angelova MI, Soleau S, Meleard P, Faucon JF, Bothorel P (1992) Preparation of giant vesicles by external fields. Kinetics and application. Prog Colloid Polym Sci 89:127-131

Bagatolli LA, Gratton E (1999) Two-photon fluorescence microscopy observations of shape changes at the phase transition in phospholipid giant unilamellar vesicles. Biophys J 77:2090-2101

Bagatolli LA, Sanchez SA, Hazlett TL, Gratton E (2003) Giant vesicles, Laurdan, and two-photon fluorescence microscopy: evidence of lipid lateral separation in bilayers. Methods Enzymol 360:481-500

Bernardino de la Serna J, Perez-Gil J, Simonsen AC, Bagatolli LA (2004) Cholesterol rules: direct observation of the coexistence of two fluid phases in native pulmonary surfactant membranes at physiological temperatures. J Biol Chem 279:40715-40722

Besenicar MP, Bavdek A, Kladnik A, Macek P, Anderluh G (2008) Kinetics of cholesterol extraction from lipid membranes by methylbeta-cyclodextrin - a surface plasmon resonance approach. Biochim Biophys Acta 1778:175-184

Brown RE (1998) Sphingolipid organization in biomembranes: what physical studies of model membranes reveal. J Cell Sci 111:1-9

Brown DA (2001) Seeing is believing: visualization of rafts in model membranes. Proc Natl Acad Sci USA 98:10517-10518

Choi Y-H, Yang C-H, Kim H-W, Jung S (2001) Molecular modeling studies of the $\beta$-cyclodextrin in monomer and dimer form as hosts for the complexation of cholesterol. J Incl Phenom Macro 39:71-76

Duchene D, Wouessidjewe D (1992) Industrial uses of cyclodextrins and their derivatives. J Coord Chem 27:223-236

Evans RW, Williams MA, Tinoco J (1987) Surface areas of 1-palmitoyl phosphatidylcholines and their interactions with cholesterol. Biochem J 245:455-462

Giocondi MC, Milhiet PE, Dosset P, Le Grimellec C (2004) Use of cyclodextrin for AFM monitoring of model raft formation. Biophys J 86:861-869

Harris JS, Epps DE, Davio SR, Kezdy FJ (1995) Evidence for transbilayer, tail-to-tail cholesterol dimers in dipalmitoyl glycerophosphocholine liposomes. Biochemistry 34:3851-3857

Hedges AR (1998) Industrial applications of cyclodextrins. Chem Rev 98:2035-2044

Ilangumaran S, Hoessli DC (1998) Effects of cholesterol depletion by cyclodextrin on the sphingolipid microdomains of the plasma membrane. Biochem J 335:433-440

Irie T, Otagiri M, Sunada M, Uekama K, Ohtani Y, Yamada Y, Sugiyama Y (1982) Cyclodextrin-induced hemolysis and shape changes of human erythrocytes in vitro. J Pharmacobiodynamics 5:741-744

Jaureguiberry MS, Tricerri MA, Sanchez SA, Garda HA, Finarelli GS, Gonzalez MC, Rimoldi OJ (2010) Membrane organization and regulation of cellular cholesterol homeostasis. J Membr Biol 234:183-194

Jouni Z, McGill B, Wells M (2002) Beta-cyclodextrin facilitates cholesterol efflux from larval Manduca sexta fat body and midgut in vitro. Comp Biochem Physiol B Biochem Mol Biol 132:699-709

Lafont F, Nhieu GTV, Hanada K, Sansonetti P, van-der-Goot FG (2002) Initial steps of Shigella infection depend on the cholesterol/sphingolipid raft-mediated CD44-IpaB interaction. EMBO J 21:4449-4457

Loftsson T, Duchêne D (2007) Cyclodextrins and their pharmaceutical applications. Int J Pharm 329:1-11

Needham D, McIntosh TJ, Evans E (1988) Thermomechanical and transition properties of dimyristoyl phosphatidylcholine/cholesterol bilayers. Biochemistry 27:4668-4673

Ohvo H, Slotte JP (1996) Cyclodextrin-mediated removal of sterols from monolayers: effects of sterol structure and phospholipids on desorption rate. Biochemistry 35:8018-8024 
Parasassi T, Gratton E (1995) Membrane lipid domains and dynamics as detected by Laurdan fluorescence. J Fluoresc 8:365-373

Parasassi TS, DeStasio G, Ravagnan G, Rusch R, Gratton E (1991) Quantitation of lipid phases in phospholipid vesicles by the generalized polarization of Laurdan fluorescence. Biophys $\mathrm{J}$ 60:179-189

Parasassi T, Stefano MD, Loiero M, Ravagnan G, Gratton E (1994) Cholesterol modifies water concentration and dynamics in phospholipid bilayers: a fluorescence study using Laurdan probe. Biophys J 66:763-768

Roux M, Auzely-Velty R, Djedaini-Pilard F, Perly B (2002) Cyclodextrin-induced lipid lateral separation in DMPC membranes: ${ }^{2} \mathrm{H}$ nuclear magnetic resonance study. Biophys $\mathrm{J}$ $82: 813-822$

Sanchez SA, Bagatolli LA, Gratton E, Hazlett TL (2002) A twophoton view of an enzyme at work: Crotalus atrox venom $\mathrm{PLA}_{2}$ interaction with single-lipid and mixed-lipid giant unilamellar vesicles. Biophys J 82:2232-2243

Sánchez SA, Tricerri MA, Gratton E (2007) Interaction of high density lipoprotein particles with membranes containing cholesterol. J Lipid Res 48:1689-1700

Sánchez SA, Tricerri MA, Ossato G, Gratton E (2010) Lipid packing determines protein-membrane interactions: challenges for apolipoprotein A-I and high density lipoproteins. Biochim Biophys Acta 1798:1399-1408

Sankaram MB, Thompson TE (1990) Modulation of phospholipid acyl chain order by cholesterol. A solid-state deuterium nuclear magnetic resonance study. Biochemistry 29:10676-10684
Sankaram MB, Thompson TE (1991) Cholesterol-induced fluid-phase immiscibility in membranes. Proc Natl Acad Sci USA 88: $8686-8690$

So PTC, French T, Yu WM, Berland KM, Dong CY, Gratton E (1995) Time resolved fluorescence microscopy using two-photon excitation. Bioimaging 3:49-63

So PTC, French T, Yu WM, Berland KM, Dong CY, Gratton E (1996) Two-photon fluorescence microscopy: time-resolved and intensity imaging. In: Wang XF, Herman B (eds) Fluorescence imaging spectroscopy and microscopy. Wiley, New York, pp 351-374

Tricerri MA, Toledo JD, Sanchez SA, Hazlett TL, Gratton E, Jonas A, Garda HA (2005) Visualization and analysis of apolipoprotein A-I interaction with binary phospholipid bilayers. J Lipid Res 46:669-678

Veatch SL, Keller SL (2003) Separation of liquid phases in giant vesicles of ternary mixtures of phospholipids and cholesterol. Biophys J 85:3074-3083

Veatch SL, Polozov IV, Gawrisch K, Keller SL (2004) Liquid domains in vesicles investigated by NMR and fluorescence microscopy. Biophys J 86:2910-2922

Weber G, Farris FJ (1979) Synthesis and spectral properties of a hydrophobic fluorescent probe: 2-dimethylamino-6-propionyl naphthalene. Biochemistry 18:3075-3078

Zidovetzki R, Levitan I (2007) Use of cyclodextrins to manipulate plasma membrane cholesterol content: evidence, misconceptions and control strategies. Biochim Biophys Acta 1768:1311-1324 